\title{
Post-transfusion survival of hydroxyethyl starch 450/0.70 in man: a long-term study
}

\author{
JOHN MILTON MISHLER, ${ }^{1}$ COLIN R RICKETTS, ${ }^{2}$ AND \\ EDWARD J PARKHOUSE ${ }^{2}$
}

From the ${ }^{1}$ Medizinische Universitätsklinik Köln, D-5000 Köln 41, FRG, and the ${ }^{2} M R C$ Industrial Injuries and Burns Unit, Birmingham Accident Hospital, Birmingham B15 INA, UK

SUMMARY Intravascular persistence concomitant with changes occurring in the circulating molecular size distribution were determined in five healthy normovolaemic men dosed with $7 \mathrm{ml} / \mathrm{kg}$ of a $6 \%$ $(w / v)$ solution of a species of hydroxyethyl starch (HES 450/0.70) possessing a $M_{w}$ of 450000 daltons combined with a molar hydroxyethyl group substitution (MS) of 0.70 (70 hydroxyethyl groups/100 glucose residues). The concentration of HES $450 / 0 \cdot 70$ in serum fell to $24 \%$ of its peak value (measured 2 minutes post injection) one week after the infusion. By 17 weeks after injection, $<1.0 \%$ remained in the intravascular space. The HES 450/0.70 material recovered from the bloodstream 2 minutes after injection was shown by gel filtration on a column of Sepharose CL-4B to be less polydisperse than the injected solution. The $\mathrm{K}_{\mathrm{av}}$ calculated for the peak of material eluted after one week showed a definite shift of molecular size toward that of a lower molecular weight composition. However, at four weeks the value of $\mathrm{K}_{\mathrm{av}}$ indicated a shift toward the high molecular weight region of the injected solution, and by seven weeks this movement was quite pronounced. These data clearly indicate the complex nature of the removal of HES 450/0.70 from the intravascular space of man and appear to substantiate previous clinical studies reporting that the MS plays the major role influencing the rate of elimination of this material from the bloodstream.

Attachment of hydroxyethyl groups through ether linkages to the hydroxyl group of the parent amylopectin molecule can effectively retard the hydrolysis mediated by $\alpha$-amylase. ${ }^{1}$ The concept of adopting such a form of hydroxyethylated amylopectin as a potential colloidal agent to augment a diminished plasma volume was later proved feasible both experimentally ${ }^{2}$ and clinically. ${ }^{3}$ From clinical studies conducted at a later date, ${ }^{4}$ a species of hydroxyethylated amylopectin (HES $450 / 0 \cdot 70$ ) possessing a weight average molecular weight $\left(\mathrm{M}_{\mathrm{w}}^{-}\right)$of 450000 daltons combined with a molar hydroxyethyl group substitution (MS) of $\mathbf{0 . 7 0}$ (70 hydroxyethyl groups/ 100 glucose residues) was shown to persist in the bloodstream in a similar manner to a dextran having a $\mathrm{M}_{\mathrm{w}}^{-}$ranging between 56000 and 70000 daltons during the initial 24 hours after dosing. Even though HES $450 / 0 \cdot 70$ has been utilised clinically for over a decade in the management of hypovolaemia, little is known of its long-term persistence in man, and especially the nature of the changes occurring

Received for publication 6 August 1979 in the circulating molecular size distribution of this material.

In some recent novel clinical uses of HES 450/0 70, such as an adjunct during centrifugal leucapheresis ${ }^{5}$ and as a cryoprotectant for platelets, ${ }^{6}$ infusion of this material serves no 'therapeutic purpose' other than to decrease the suspension stability of blood in normal donors in the former case, and as a cryoprotectant colloid in the latter application. In both these clinical situations information as to the elimination of HES $450 / 0.70$ in normal man appears to be rather important. We have, therefore, endeavoured to document, during a long-term investigation in normal man, the persistence of HES $450 / 0 \cdot 70$ in blood concomitantly with determining the changes taking place in the molecular size composition of material remaining in the intravascular space.

\section{Material and methods}

SUBJECTS AND PROCEDURES

Five normovolaemic healthy men (aged 21 to 26) 
comprised the study group. Their body surface areas varied from 1.61 to $1.89 \mathrm{~m}^{2}$, which corresponded approximately to whole blood volumes of 4025 to $4725 \mathrm{ml}$. The experiment was thoroughly explained to each subject and their consent was obtained.

A standardised dose $(7 \mathrm{ml} / \mathrm{kg})$ of a $6 \%(\mathrm{w} / \mathrm{v})$ solution of HES $450 / 0 \cdot 70\left(M_{w}^{-} 450000\right.$ daltons, number average molecular weight $\left(M_{n}^{-}\right) 71000$ daltons, $M_{w}^{-} / M_{n}^{-}=6 \cdot 3$, intrinsic viscosity $0.25 \mathrm{dl} / \mathrm{g}$, MS: 70 hydroxyethyl groups/100 glucose residues, Dr E Fresenius KG, FRG) in $0.9 \%$ isotonic saline was infused through a 19-gauge cannula inserted in a forearm vein over a 26 - to 48 -minute period (mean rate of injection was $0.20 \pm 0.05$ (SD) $\mathrm{ml} / \mathrm{kg} /$ min). Samples of whole blood were collected into sterile plastic syringes (without anticoagulant) before (baseline) and 2 minutes after the injection. Samples were also taken 1, 2, 4, 7, 10, and 17 weeks after dosing. The samples of whole blood were allowed to clot in a blood bank refrigerator maintained at between 4 and $6^{\circ} \mathrm{C}$. Serum was separated from the clotted sample within 45 minutes of collection by centrifugation at $2500 \times g$ (Sorvall RC2-B centrifuge, USA) for 10 minutes at $4^{\circ} \mathrm{C}$.

\section{LABORATORY DETI RMINATIONS}

After precipitation o $i$ the serum proteins with $5 \%$ $(w / v)$ trichloroacetic acid (TCA), total concentration of carbohydrate was assayed in triplicate by the anthrone method. ${ }^{7}$ The difference between the concentration of total carbohydrate and serum glucose (measured by the hexokinase $\operatorname{method}^{8}$ ) at each sampling interval constituted the concentration of HES $450 / 0 \cdot 70$.

GEL FILTRATION

In determining the molecular size distribution of HES 450/0.70 remaining in the bloodstream after dosing, TCA filtrates of serum were concentrated by ultrafiltration and dialysed in Cellophane tubing (diameter $6.2 \mathrm{~mm}$ and a pore size of $<7.2 \mathrm{~nm}$ ), and the resulting concentrate was applied to the gel filtration column. A total of $50 \mathrm{mg}$ of HES $450 / 0 \cdot 70$ (a volume of serum containing $10 \mathrm{mg}$ of HES $450 / 0 \cdot 70$ from each of the five subjects was pooled, thereby avoiding weighting the results according to concentration) was used at each interval of sampling to provide sufficient material for analysis. Gel filtration was done on a column of Sepharose CL-4B (Pharmacia Fine Chemicals, Sweden) in $M / 15$ sodium phosphate buffer ( $\mathrm{pH} 7 \cdot 5,1 \mathrm{vol}$ ) plus $0.9 \%$ isotonic saline $(9 \mathrm{vol})$, as previously described. ${ }^{9}$ The rate of elution ranged between 8.9 and $9.2 \mathrm{ml} / \mathrm{h}$, and $5 \mathrm{ml}$ fractions of elute were collected as required. The effluent solution was continuously monitored by a differential refractometer (Waters Model R4, USA) operating a chart recorder. In some cases the material contained in each peak of eluted solution was ultrafiltrated, dialysed, and freeze-dried. The solid material so recovered was stained with Lugol's iodine solution in order to test for the presence of carbohydrate. Recorder reading was found to be linear with HES $450 / 0 \cdot 70$ concentration over a range of $0-1 \mathrm{mg} / \mathrm{ml}$, corresponding with a $5-50$ millivolt reading. All peaks eluted from the column were within this range of concentration. The column was calibrated with Blue Dextran for void volume $\left(\mathrm{V}_{0}\right)$ and sodium iodide for total volume $\left(V_{t}\right)$. The $K_{a v}$ for each of the eluted peaks was calculated according to the formula $\left(\mathrm{V}_{\mathrm{e}}=\right.$ elution volume):

$$
K_{a v}=\frac{V_{e}-V_{0}}{V_{t}-V_{0}} .
$$

\section{Results}

INTRAVASCULAR CONCENTRATION OF HES $450 / 0 \cdot 70$

In five normal subjects dosed with an average of $449 \pm 26$ (SD) $\mathrm{ml}$ of a $6 \%$ solution of HES $450 / 0 \cdot 70$, the concentration of this material in the bloodstream fell to $24 \%$ of its peak value (measured 2 minutes after the termination of the infusion) one week after injection (Fig. 1). By 17 weeks after infusion $<1.0 \%$ remained in the intravascular space.

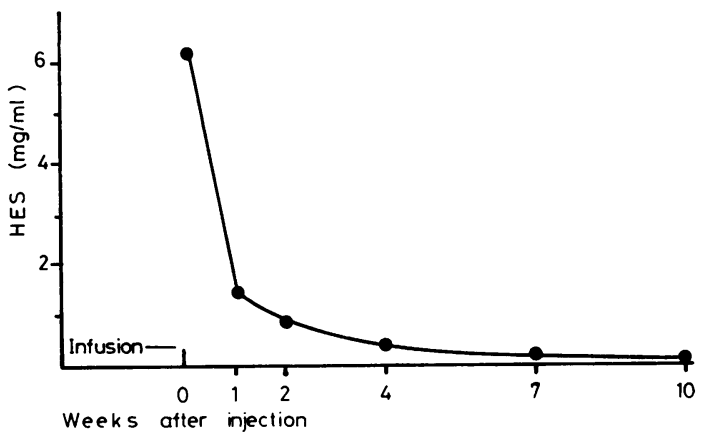

Fig. 1 Concentration of HES 450/0.70 in serum of five normal men dosed with $7 \mathrm{ml} / \mathrm{kg}$ of a $6 \%(\mathrm{w} / \mathrm{v})$ solution of this material. Each point represents the mean of five determinations. The average concentration of HES $450 / 0 \cdot 70$ was $6 \cdot 12 \pm 0.51$ (SD) $\mathrm{mg} / \mathrm{ml}$ immediately (2 min) after injection. The intravascular concentration then fell progressively to $1 \cdot 51 \pm 0 \cdot 16$, $0.96 \pm 0.12,0.49 \pm 0 \cdot 13,0 \cdot 22 \pm 0.05$, and $0.15 \pm 0.02$, $\mathrm{mg} / \mathrm{ml}$ one, two, four, seven, and 10 weeks after the infusion. The concentration of HES $450 / 0.70$ detected in the serum after 17 weeks was $0.06 \pm 0.01 \mathrm{mg} / \mathrm{ml}$, which represented $<1.0 \%$ of the peak concentration measured 2 min after injection. 


\section{CHANGES IN MOLECULAR COMPOSITION} OF HES $450 / 0 \cdot 70$

Changes that had taken place in the molecular size distribution of circulating HES 450/0.70 determined at various intervals after dosing are shown in Figure 2. As can be readily observed, the molecular composition of the injected solution is made up of a wide distribution (polydispersion) of various molecular weight size material, as would be predicted by the ratio of $\mathbf{M}_{\mathbf{w}}^{-} / \mathbf{M}_{\bar{n}}^{-}$. However, 2 minutes after the infusion of HES $450 / 0.70$ the recovered material is of a narrower size distribution relative to the injected solution. The $\mathrm{K}_{\text {av }}$ calculated for the peak of material eluted after one week showed a definite shift of molecular size toward that of a lower molecular weight composition (Table). However, four weeks after the infusion the value of $K_{a v}$ indicated a shift toward the high molecular weight region of the injected solution, and by seven weeks this movement was quite pronounced. The solid material recovered from the peaks of eluate obtained after four and seven weeks stained a reddish-brown colour with Lugol's iodine solution in the same manner as the HES 450/0.70 contained in the injected solution, confirming the presence of carbohydrate in these peaks.

There was no significant difference between the infrared spectrum of HES $450 / 0 \cdot 70$ recovered from

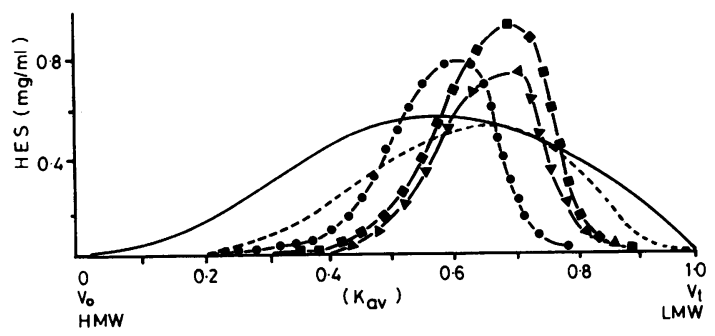

Fig. 2 Changes in molecular composition of HES $450 / 0 \cdot 70$ after the dosing of five normal men with $7 \mathrm{ml} / \mathrm{kg}$ of a $6 \%(\mathrm{w} / \mathrm{v})$ solution. Gel filtration on a column of Sepharose CL-4B of $50 \mathrm{mg}$ of HES 450/0.70 of $M_{\bar{w}}^{-} 450000$ daltons (-) and a similar quantity (pooled) of HES 450/0.70 recovered from serum $2 \mathrm{~min}$ after injection (-- - ) and one ( $-\square)$, four ( $\left.\boldsymbol{\Delta}_{-} \boldsymbol{-}\right)$ ), and seven (O-O) weeks after dosing. In general, the distribution of molecular size present in the bloodstream is less polydispersed than was observed in the injected material. After one week there is a definite shift of molecular size towards that of a lower molecular weight (LMW) composition. However, at four weeks there are signs of a shift toward the high molecular weight $(H M W)$ region of the injected solution, and by seven weeks this movement is quite pronounced. The calculated value for the $K_{a v}$ of each eluted peak is given in the Table.
$K_{a v}$ values for elution peaks* obtained after separation of various molecular weight HES $450 / 0 \cdot 70$ material on a column of Sepharose CL-4B (see Material and methods).

\begin{tabular}{|c|c|c|c|c|c|}
\hline & \multirow{2}{*}{$\begin{array}{l}\text { Injected } \\
\text { HES } 450 / 0 \cdot 70 \\
\text { solution }\end{array}$} & \multirow{2}{*}{$\begin{array}{l}\text { Immediately } \\
\text { after } \\
\text { injection }\end{array}$} & \multicolumn{3}{|c|}{ Weeks after injection } \\
\hline & & & 1 & 4 & 7 \\
\hline $\mathbf{K}_{\mathrm{av}}$ & 0.57 & 0.67 & 0.70 & 0.68 & 0.60 \\
\hline
\end{tabular}

*The peaks were obtained from samples of pooled serum and correspond to the elution profiles in Figure 2.

the infused solution and the spectra of material recovered from the plasma four and seven weeks after dosing.

\section{Discussion}

Amylopectin should be acceptable as a therapeutic colloid when infused into man, based on its similarity to normal body glycogen. Ordinary solutions of starch, however, are physically unstable and are rapidly catabolised in the blood of rabbits ${ }^{10}$ and canines $^{11}$ after intravenous dosing. On the other hand, physically stable branched polymers of starch, such as amylopectin, can be reacted with ethylene oxide in an alkaline environment, ${ }^{1}$ enabling hydroxyethyl groups to be attached by ether linkages to the hydroxyl group of the glucose residue. Hydroxyethylation may attach the hydroxyethyl group on either the 2-, 3-, or 6-carbon of the glucose residue, thus effectively retarding the hydrolysis mediated by $\alpha$-amylase contained in blood. ${ }^{12} 13$ In the clinically available species of HES, approximately $80 \%$ of the substitution of the hydroxyethyl group would be expected to take place on the 2-carbon. ${ }^{14}$ The predominant substitution of the hydroxyethyl group on the 2-carbon appears constant up to an MS of 1.4. This 2-carbon hydroxyl group is more acidic relative to those at either the 3-carbon or 6-carbon positions. The difference in acidity is caused by the electronic effects of the oxygen atoms. ${ }^{14}$ Another factor in this selective substitution on the 2-carbon is the possibility of stabilisation of the negative alkoxylion at either the 2-carbon or 3-carbon position by the proton of the adjacent hydroxyl group.

Of interest clinically is the observation that the actual position of the hydroxyethyl group, whether it be on the 2-, 3-, or 6-carbon of the glucose residue, appears to exert a significant influence on retardation of $\alpha$-amylase catabolism. ${ }^{13} \mathrm{~A}$ diminished rate of hydrolysis by $\alpha$-amylase is reported with substitution on the 2-carbon, or with multiple hydroxyethylations on the same carbon. However, less resistence to hydrolysis is reported to occur when the 2-carbon/6- 
carbon substitution ratio is below 2.4. Effective retardation of $\alpha$-amylase attack implies that hydroxyethylation on any glucose residue within a specific five-unit amylose substrate must deviate from the accepiable pattern of substitution. ${ }^{15}$ For example, substitution on the 2-carbon of glucose-1, -3 , or -5 in the five-unit amylose substrate (reducing end is glucose-1) would significantly hinder attack by $\alpha$-amylase.

In canine ${ }^{11}$ or rabbit ${ }^{12}$ models, the intravascular half-lives $\left(\right.$ IT $\left._{50}\right)$ of various injected species of HES can be prolonged at any given $\mathrm{M}_{\overline{\mathrm{w}}}$ by increasing the MS, or, conversely, at any given MS increasing the $\mathbf{M}_{\mathbf{w}}^{-}$will impede clearance of this material from the circulation. In these studies, the MS was below 1.4, indicating that the substitution of the hydroxyethyl group took place in the majority of instances on the 2-carbon. In man, the actual persistence of any species of HES does not depend wholly on the relative molecular weight size distribution $\left(\mathbf{M w}_{\mathbf{w}} / \mathbf{M}_{\mathbf{n}}^{-}\right)$ of the injected solution but rather on the degree of MS.416-18 The clearance from the intravascular space during the initial hour after injection does depend on the $\mathbf{M}_{\mathbf{w}}^{-}$(more accurately on a subpopulation of molecules characterised by the $\mathbf{M}_{\mathbf{n}}^{-}$), but after this initial phase of rapid elimination the MS plays the major role, influencing catabolism and subsequent excretion through the kidneys. For example, given as a single bolus injection, the clinical IT $_{\overline{5} 0 S}$ of HES $350 / 0 \cdot 60,19$ HES $264 / 0 \cdot 43,18$ and HES $150 / 0 \cdot 70^{17}$ are approximately 12,3 , and 10 hours, respectively. In normal man, dosed on a single occasion with HES $450 / 0 \cdot 70$, the IT $_{50}$ was reported to range between 24 and 28 hours. ${ }^{416} 20 \mathrm{Up}$ to 72 hours after a single intravenous dose the disappearance of HES 450/0.70 from the bloodstream can be described mathematically and appears to consist of three phases of elimination (rapid, intermediate, and slow IT ${ }_{50}$ s). ${ }^{4}$ Mathematically speaking, HES 450/0·70 is eliminated from the blood in a similar fashion for up to 24 hours each day after the infusion of this material on three consecutive days. ${ }^{21}$ However, the actual long-term persistence of HES $450 / 0 \cdot 70$ in blood appears to differ significantly from predictions made by mathematical models describing elimination over a relatively short period of observation..$^{20}$ In normal subjects injected with $60 \mathrm{~g}$ on a single occasion $^{4}$ or $30 \mathrm{~g}$ on each of three consecutive days (total $90 \mathrm{~g}$ ), ${ }^{21}$ the $1.0 \%$ level of remaining HES 450/0.70 material in the blood was mathematically predicted to occur 2.3 and 4.5 weeks after dosing, respectively. In a previous long-term study, ${ }^{20}$ the $1.0 \%$ concentration of HES $450 / 0.70$ in serum was not achieved even after 17 weeks. However, in the present study the concent ation of HES 450/0.70 in serum fell to less than $1.0 \% 17$ weeks after injection (Fig. 1). The discrepancy between the disappearance of HES 450/0.70 from blood as predicted mathematically and what is actually observed may reside in the character of the residual material remaining after the rapid and intermediate phases of elimination. If the injected solution of HES $450 / 0 \cdot 70$ contains molecules in a small fractionated range combined with a homogenous pattern of hydroxyethylation, one assumes that disappearance could be predicted adequately by an equation of the first order. However, up to 72 hours after injection elimination is predicted only by an exponential function. ${ }^{41}$ These data clearly indicate the complex nature of the clearance of this material from the circulation.

The interplay between $\mathbf{M}_{\mathbf{w}}^{-}\left(\mathbf{M}_{\mathbf{n}}^{-}\right)$and MS during the slower phase(s) of elimination has been characterised by investigating the changes taking place in the molecular weight size composition of the residual material. In rabbits infused once with either HES $150 / 0 \cdot 70$ or HES $450 / 0 \cdot 70$, the Sepharose CL-4B elution profile of residual material recovered 24 hours after njection indicated the presence of an intermediate fraction occupying a position between the low and high molecular weight regions of the injected solution. ${ }^{9}$ In man, a similar intermediate fraction is recovered from blood three weeks after the third and final consecutive injection of HES $450 / 0 \cdot 70 .{ }^{22}$ In the present study, a definite intermediate fraction was observed seven weeks after a single dose of this material was administered (Fig. 2; Table). However, in normal man infused once with a species of HES (HES 264/0.43) in which the MS was reduced $40 \%$, only smaller molecular weight size material relative to the injected solution was recovered. ${ }^{23}$ The appearance of an intermediate fraction after dosing with HES 450/0.70 may imply that a condition of steady-state hydrolysis had occurred in which the rate of catabolism of the low and high molecular weight fractions contained in the injected solution are similar. On the other hand, the continual movement of the molecular weight size distribution toward the low molecular weight region of the injected HES 264/0.43 solution suggests that catabolism of the higher molecular weight fraction contained in the injected solution may occur at a rate greater than the degradation of the low molecular weight fraction. It is known that as the MS approaches 1.0, the number of di-, tri-, and tetra-substituted residues of glucose becomes greater, ${ }^{14}$ thus increasing the likelihood that unfavourable patterns of substitution will be incorporated into any five-unit amylose substrate. ${ }^{15}$ The data contained in the present study and that previously published suggest that higher degrees of MS increase the number of multiple substitutions, thus retarding 
to a greater extent the attack mediated by $\alpha$-amylase. Low degrees of MS may increase the likelihood that only mono-substitution will occur, making attack by $\alpha$-amylase more predictable.

Portions of this study were supported by the Alexander von Humboldt Foundation, the SFB 68 of the 'Deutsche Forschungsgemeinschaft', and the Medical Research Council. JMM is currently an Alexander von Humboldt Foundation Senior Research Fellow. We thank Dr JK Brown, Department of Chemistry, University of Birmingham for interpretation of the infrared spectra.

\section{References}

${ }^{1}$ Ziese W. Beitrag zur Spezifität der Amylasen. Einwirkung von Amylasen auf Oxyäthylstärke. Hoppe Seylers z Physiol Chem 1935; 235: 235-45.

${ }^{2}$ Wiedersheim M. An investigation of oxyethylstarch as a new plasma volume expander in animals. Arch int Pharmacodyn 1957; 111 : 353-61.

${ }^{3}$ Solanke T F. Clinical trial of $6 \%$ hydroxyethyl starch (a new plasma expander). Br Med J 1968; 3: 783-5.

${ }^{4}$ Metcalf W, Papadopoulos A, Tufaro R, Barth A. A clinical physiologic study of hydroxyethyl starch. Surg Gynec Obstet 1970; 131 : 255-67.

${ }^{5}$ Mishler J M. Hydroxyethyl starch as an experimental adjunct to leukocyte separation by centrifugal means: Review of safety and efficacy. Transfusion 1975; 15: 449-60.

${ }^{6}$ Choudhury C, Gunstone M J. Freeze preservation of platlets using hydroxyethyl starch (HES): A preliminary report. Cryobiology 1978; 15: 493-501.

'Brake J M. Research Laboratory Technique $N^{0} 176$ and 177. McGraw Laboratories, Irvine, California, USA 1966.

${ }^{8}$ Schmidt F H. Die enzymatische Bestimmung von Glucose und Fructose nebeneinander. Klin Wschr 1961; 39: 1244-7.

${ }^{9}$ Farrow S P, Hall M, Ricketts C R. Changes in the molecular composition of circulating hydroxyethyl starch. Br J Pharmac 1970; 38: 725-30.

${ }^{10}$ Terashima T. Nutritional significance of the parenteral administration of polysaccharides. Jap J Gastroenterol 1937; 9: 273-85.

${ }^{11}$ Thompson W L, Britton J J, Walton R P. Persistence of starch derivatives and dextran when infused after hemorrhage. J Pharmac Exper Therap 1962; 136: 125-32.
${ }^{12}$ Tamada $T$, Okada $T$, Ishida $R$, Irikura $T$. Studies on hydroxyethyl starch as a plasma volume expander. I. Persistence and enzyme resistance of HES. Pharmacometrics 1970; 4: 505-12.

${ }^{13}$ Yoshida M, Yamashita T, Matsuo J, Kishikawa T. Enzymatic degradation of hydroxyethyl starch. I. Influence of the distribution of hydroxyethyl groups on the enzymic degradation of hydroxyethyl starch. Die Stärke 1973; 25: 373-6.

${ }^{14}$ Merkus H G, Mourits J W, Degalen L, Dejong W A. Substitution distribution in hydroxyethyl starch. Die Stärke 1977; 29: 406-11.

${ }^{15}$ Chan Y C. 0-(2-hydroxyethyl)-amylose as the substrate of porcine pancreatic $\alpha$-amylase action: Structural analysis of 0-(2-hydroxyethyl) maltooligosaccharides. PhD thesis, Iowa State University, Iowa, USA, 1975.

${ }^{16}$ Mishler J M, Borberg $\mathbf{H}$, Emerson $\mathbf{P} \mathbf{M}$, Gross R. Hydroxyethyl starch: An agent for hypovolemic shock treatment. I. Serum concentrations in normal volunteers following three consecutive daily infusions. J Surg Res 1977; 23: 239-45.

${ }^{17}$ Mishler J M, Parry E S, Petrie A. Plasma clearance and renal excretion of erthrocyte cryoprotectant hydroxyethylated amylopectin. Br J Haematol 1978; 40: 231-7.

${ }^{18}$ Mishler J M, Parry E S, Sutherland B A, Bushrod J R. A clinical study of low molecular weight-hydroxyethyl starch, a new plasma expander. Br J Clin Pharmac 1979; $7:$ 619-22.

${ }^{19}$ Mishler J M. The plasma kinetics of hydroxyethyl starch 350/0-60. A potential new adjunct for centrifugal leucapheresis. Am J Hematol 1980; in press.

${ }^{20}$ Boon J C, Jesch F, Ring J, Messmer K. Intravascular persistence of hydroxyethyl starch in man. Eur Surg Res 1976; 8: 497-503.

${ }^{21}$ Mishler J M, Beez M. Die mathematische Beschreibung der intravasalen Ausscheidung von HÄS nach wiederholten Infusionen beim Menshen. Infusionsther Klin Ernacht 1979; 6: 119-22.

${ }^{22}$ Mishler J M, Ricketts C R, Parkhouse E J. Changes in the molecular composition of circulating hydroxyethyl starch following consecutive daily infusions in man. Br J Clin Pharmac 1979; 7 : 505-9.

${ }^{23}$ Mishler J M, Ricketts C R, Parkhouse E J, Borberg H, Gross R. Catabolism of low molecular weighthydroxyethylated amylopectin in man. I. Changes in the circulating molecular composition. J Lab Clin Med 1980; in press.

Requests for reprints to: Dr John Milton Mishler, IV, Medizinische Universitätsklinik Köln, Joseph-StelzmannStrasse 9, D-5000 Köln 41, FRG. 